\title{
Design and Characterization of PCB Spiral Coils for Inductive Power Transfer in Medium Voltage Applications
}

\author{
X. Du, C. Li, and D. Dujic
}

This material is posted here with permission of the IEEE. Such permission of the IEEE does not in any way imply IEEE endorsement of any of EPFL's products or services. Internal or personal use of this material is permitted. However, permission to reprint / republish this material for advertising or promotional purposes or for creating new collective works for resale or redistribution must be obtained from the IEEE by writing to pubs-permissions@ieee. org. By choosing to view this document, you agree to all provisions of the copyright laws protecting it. 


\title{
Design and Characterization of PCB Spiral Coils for Inductive Power Transfer in Medium Voltage Applications
}

\author{
Xiaotong Du, Student Member, IEEE, Chengmin Li, Member, IEEE, and \\ Drazen Dujic, Senior Member, IEEE
}

\begin{abstract}
Coil design and operating frequency are key factors impacting the efficiency of inductive power transfer system. This paper presents the modeling of PCB spiral coils, design optimization and analysis on coil geometry and operating frequency for medium voltage applications, such as auxiliary power supplies for medium voltage converters. Analytic model considering the geometric distribution of the magnetic field is proposed to calculate with high accuracy equivalent electrical parameters of coils. For a defined design space and constraints, a large number of coils are designed in order to determine their performances and select optimal coil geometries. Selected coil designs are prototyped and experimentally verified under design-specific conditions. In addition, further characterization is performed considering variations of several parameters that can be encountered in practice.
\end{abstract}

Index Terms-Medium Voltage Converter, Inductive Power Transfer, PCB Spiral Coil, Modeling, Magnetic Field Distribution

\section{INTRODUCTION}

Inductive power transfer (IPT) is a widely used technology to transfer power without physical contact in the near field. Comparing to another near field wireless power transfer technology: capacitive power transfer (CPT) which transfers power by electrostatic induction, IPT relying on the power transfer by magnetic induction via the electromagnetic field, can transfer power through wider physical gaps [1].

IPT technology has been applied as the power supply in various applications spanning a wide power range and a large distance range. In an IPT application, physical coil size and gap distance are the most dominant figures that limit output power and efficiency. Applications characterized by very low power demand $(<1 \mathrm{~W})$ such as bio-medical implants [2] usually have a tiny size $\left(<1 \mathrm{~cm}^{2}\right)$ and typically small gap $(<3$ $\mathrm{cm})$ [3][4]. These size constraints require the IPT system to operate at $\mathrm{MHz}$ range. High power level $(>1 \mathrm{~kW})$ applications such as charging of electric vehicles (EV) [5] have relaxed restriction on coil size $\left(>10 \mathrm{dm}^{2}\right)$ and operating frequency is usually in the range of several tens of $\mathrm{kHz}$. In the middle power range $(1 \mathrm{~W}$ to $1 \mathrm{~kW})$, applications include portable devices, household appliances, special industrial and mechatronics applications. Among these applications in medium power range, the existing researches are normally focused on the power transfer over a short gap, due to low voltages involved [6].

The autors are with the Power Electronics Laboratory, École Polytechnique Fédérale de Lausanne (EPFL), 1015 Lausanne, Switzerland (e-mail: xiaotong.du@epfl.ch; chengmin.li@epfl.ch; drazen.dujic@epfl.ch)
Considering application of auxiliary power supply in medium voltage (MV) converters, mechanical arrangement imposes constraints on the coil size, and a large gap is typically required due to MV insulation coordination [7], [8]. Due to those restrictions of this application scenario, the large gap $(>50 \mathrm{~mm})$ and small coil size $\left(<10 \mathrm{dm}^{2}\right)$ represent the design constraint as well as challenge for the design of IPT system.

In an IPT system, the performance of coils is one of the factors that dominate the performance of the IPT system.

IPT coil could be in either planar form (2D) or threedimensional structure (3D). 3D structures are usually used in multi-transmitter or multi-receiver IPT systems [9] [10] operating at $\mathrm{MHz}$ range. In the single-transmitter and singlereceiver system, planar coil structures are most widely used [11].

The geometry of the planar coil could be rectangular, double-D (DD), or circular. The DD shape is widely used in EV [12] because it has better performance against lateral displacement but shows higher power loss than the single loop coils [13]. For applications where coils are installed in fixed position, the lateral misalignment is not a severe problem. Therefore, DD coil is not the optimal choice. Comparing circular spiral coils with polygonal spiral coils, the former provides better coupling within the same surface area [14] and higher optimal quality factor [15].

The circular spiral coil could be realized with Litz wire [16] or copper trace on PCB. PCB tracks have much sharper edges and could challenge the dielectric design. However, PCB based IPT as shown in Fig. 1 prevails in consistency of parameters during manufacturing and easy installation. Even though there is plenty of analysis on PCB coil performance, PCB coil's potential in transferring low amount of power (e.g. $150 \mathrm{~W}$ ) over the large gap using small size coils power in MV applications is not fully explored. There are already preliminary works on PCB coil design, including inductance, resistance and insulation. For example, self inductance is modeled with current sheet method in [17] which has large error when space between traces is much larger than trace width. AC resistance model is given in [18] which needs FEM simulation to obtain magnetic field. In [16] authors have studied on the insulation with air while in [19] insulation has been analyzed with solid dielectric materials including FR4, Polyesterimide and Teflon. However, no systematic design approach for PCB coils could be identified.

This paper's main purpose is to present a systematic 


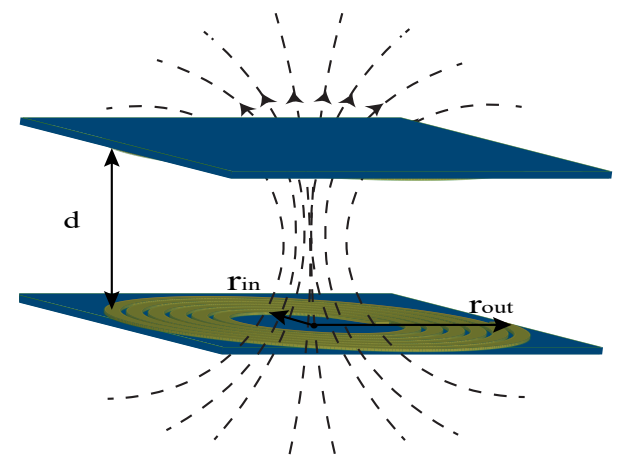

Fig. 1. An example of using PCB spiral coils to realize inductive powe transfer through air. Primary coil and secondary coil are placed face to face. $d$ is the air gap between the primary coil and secondary coil. $r_{\text {in }}$ and $r_{\text {out }}$ are the inner and outer radius of primary coil. The secondary coil could have a different geometry from the primary coil.

and generalized model-based design approach that outputs a database and from which users could select coils based on their own needs and preference. The optimization approach offers several insights in designing IPT coils and considering air (at least in present work) as the only dielectric material between transmitter and receiver. Firstly, the optimization approach explores the system performance in a wide range of coil geometries and the trade-off between the power density and efficiency is clearly demonstrated. Secondly, the influence of operating frequency on the performance of the coil pairs is shown, which permits selecting an optimal frequency, for the further system design. Finally, for the selected and prototyped coils, the limits of coil geometry to transfer a certain amount of power are determined through experimental characterization considering variation of distance between the coils as well as minor misalignment that would occur in practice. The complete IPT system would naturally include primary and secondary side power electronics stages, however optimization of these is outside the scope of this paper.

This paper is organized as follows: In Section II, design space considering specifications of typical medium voltage application is defined. In Section III, an analytic model considering the magnetic field's geometric distribution is proposed to calculate the coil's equivalent electric parameters with high speed and high accuracy. FEM simulations using ANSYS Maxwell are used for validation of modeling. In Section IV, large number of coils are designed over the design space and their performances are explored considering defined constraints. In Section V, experimental results are presented, obtained from the selected and prototyped coils. Section VI presents further performance characterization results of the same coils under extended operating condition range. Finally, Section VII concludes the paper and provides the discussion on the presented results.

\section{IPT DESIGN SPACE}

An inductive power transfer system is usually composed of compensation networks, transmitter coil, and receiver coil. The optimization of the coil geometry in an IPT system is depending on the operation mode of the compensation network.
TABLE I: Design specifications

\begin{tabular}{||ccccc||}
\hline$V_{\text {in }}$ & $V_{\text {out }}$ & $U_{\text {ins-rms }}$ & $P_{\text {out }}$ & thickness \\
\hline $80 \mathrm{~V}$ & $36 \pm 2 \mathrm{~V}$ & $6 \mathrm{kV}$ & $150 \mathrm{~W}$ & $70 \mu \mathrm{m}$ \\
\hline
\end{tabular}

Various compensation networks are proposed for different application needs, including simple compensation (SeriesParallel, Series-Series, Parallel-Parallel, Parallel-Series) and hybrid compensation networks (LCL, LLC, etc.) [20]. Choice of compensation network leads to different transfer ratios and therefore different optimization results.

The optimization approach proposed in this paper is generalized that holds the same workflow for different compensation strategies. The different typologies and inputs influence only the filtering criteria. In this paper, LCL-S compensation network as shown in Fig. 2 is considered [21].

The optimization approach is also based on the specific electric inputs and outputs of one application scenario. In this paper, the electric outputs are set for auxiliary power supply considering the MV converters application case. While the general medium voltage range up to $36 \mathrm{kV}$ is considered as relevant, $6 \mathrm{kV}$ system working voltage is taken as the case in this paper to illustrate the optimization approach. The insulation distance is defined based on this voltage from the standard IEC 61800-5-1 [22]. Other electric specifications used for the application considered in this paper are listed respectively in Table I. Output DC voltage of $36 \pm 2 \mathrm{~V}$ is defined as reference which could be further regulated with additional converters for supplying various auxiliary voltages in MV converters. Similarly, $150 \mathrm{~W}$ output is considered as reasonable maximum power level demand for one power electronics building block in MV converters, including gate drivers, controllers, cooling fan etc.

Considering the trade off between the low switching loss on power electronics switches and high efficiency on coil links, operating range is taken from $50 \mathrm{kHz}$ to $1 \mathrm{MHz}$. Considering the mechanical constraints in typical MV converter assembly, maximum coil diameter is restricted to $150 \mathrm{~mm}$. The geometries range studied in this paper are shown in Table II.

It should be noted that both the electric specifications and coil geometries restriction should be changed according to application requirements. Nevertheless, the proposed analytic model and optimization approach is generalized and can be adapted in other application scenarios.

\section{PCB SPIRAL COIL MODELING}

An accurate mathematical model of inductance and resistance is the basis for designing coils. In this section, the

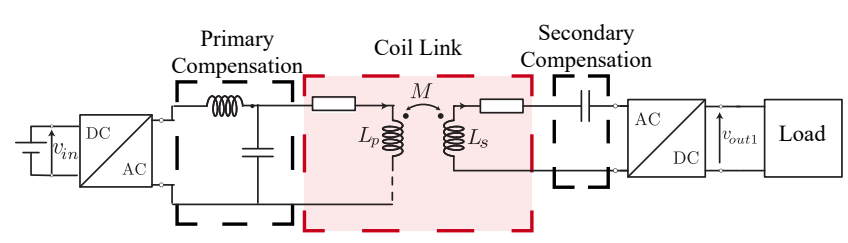

Fig. 2. Structure of auxiliary power supply for medium voltage converters Multiple series connected coils are usually used but only one pair of coil is shown for the illustration. 
TABLE II: Design space of PCB coil geometries and operating frequency

\begin{tabular}{||lccc||}
\hline Sweeping inputs & Characteristics & Value/Range & Units \\
\hline \hline Outer radius & $r_{\text {outp }}, r_{\text {outs }}$ & $10-75$ & $\mathrm{~mm}$ \\
\hline Copper trace width & $w_{p}, w_{s}$ & $1-20$ & $\mathrm{~mm}$ \\
\hline $\begin{array}{l}\text { Space between adjacent } \\
\text { copper turns }\end{array}$ & $s_{p}, s_{s}$ & $1-20$ & $\mathrm{~mm}$ \\
\hline Number of turns of one coil & $n_{p}, n_{s}$ & $\max =\frac{\text { Rout }}{w+s}$ & - \\
\hline Operating frequency & $\mathrm{f}$ & $50-1000$ & $\mathrm{kHz}$ \\
\hline
\end{tabular}

magnetic field is first mathematically modeled, followed by the modeling of self-inductance, AC resistance, and mutual inductance.

\section{A. Modeling of external magnetic field}

One of the common methods used in solving magnetic fields is to solve Maxwell's equations for rectangular cross sections by dealing with numerical integration [23]. Despite being slow and complicated, this method gives precise results. Another popular approach is the filament method, which considers coil as a dimensionless filament of negligible cross-section [24]. It gives a closed-form equation but has larger errors when the cross-sectional dimension is large. Here, a modified filament method is proposed, using two current loops on conductor edges to represent the current in one turn circular conductor. The proposed method intends to have smaller errors while keeping the calculation's simplicity.

The cross section of PCB based spiral coil is illustrated in Fig. 3. For simplicity, coaxial circles are typically used instead of spiral structure [19][25]. The external magnetic field $H_{e x}$ in the $i^{t h}$ turn defined as $H_{e x, i, t o t}$ refers to the magnetic field caused by current in other turns excluding the magnetic field caused by its own current [26].

$$
H_{e x, i, t o t}=\sum_{k=1}^{N} H_{i, k}, k \neq i
$$

Three simplifications are made in obtaining an analytic model. Firstly, since the thickness of the coil $t$ is rather small (commonly used PCB thickness is $35 \mu \mathrm{m}$ or $70 \mu \mathrm{m}$, the external $\mathrm{H}$ field parallel along the $e_{x}$ axis is ignored, and only the magnetic field in $e_{z}$ direction is considered. Secondly, since in the defined frequency range $(50 \mathrm{kHz}-1 \mathrm{MHz})$, the skin depth is at least three times smaller than trace width in the design space $(\geq 1 \mathrm{~mm})$, current in one trace is simplified to two dimensionless current loops at left and right edges. Thirdly, the nonuniform distribution of magnetic field inside the track is simplified to be constant and is represented by the magnetic field at the center point of the track. Taking $n^{\text {th }}$ turn as an example, the average magnetic field in $i^{\text {th }}$ turn caused by $n^{\text {th }}$ turn, as shown in Fig. 3 is [26]:

$$
\begin{aligned}
H_{e x, i, n} & =\frac{1}{\mu_{0}} \frac{\mu I_{l}}{4 \pi} \frac{1}{r_{n-l}+r_{i}}\left[K\left(k_{l}\right)+\frac{r_{n-l}^{2}-r_{i}^{2}}{\left(r_{n-l}-r_{i}\right)^{2}} E\left(k_{l}\right)\right] \\
& +\frac{1}{\mu_{0}} \frac{\mu I_{r}}{4 \pi} \frac{1}{r_{n-r}+r_{i}}\left[K\left(k_{r}\right)+\frac{r_{n-r}^{2}-r_{i}^{2}}{\left(r_{n-r}-r_{i}\right)^{2}} E\left(k_{r}\right)\right]
\end{aligned}
$$

In the equation above, $I_{l}$ and $I_{r}$ are the current on the left and the right edge, respectively. $K(k)$ and $E(k)$ are complete elliptical integrals of first and second kind to modulus $k$.

$$
k_{l / r}^{2}=\frac{4 r_{n-l / r} r-i}{(R+p)^{2}}
$$

In order to get $I_{l}$ and $I_{r}$, two steps are followed. Firstly, currents are supposed to be equally concentrated on the left and the right edge $I_{l}=I_{r}=\frac{I}{2}$, where $I$ is the total current inside the conductor. Initial magnetic field is obtained with (2). The electric field distribution along $\mathrm{x}$ axis is

$$
\frac{\partial E_{y}}{\partial x}=-j \omega \mu H
$$

Further, $J_{\text {prox-edge }}$, the current density at both edges under the external magnetic field is obtained

$$
\left|J_{\text {prox-edge }}\right|=-j \omega \mu \frac{w}{2} \sigma H
$$

In this equation, $\omega$ is the angular frequency of current excitation, $\mu$ is the magnetic permeability of copper which is $4 \pi e^{-7}$, $w$ is the width of copper trace, $\sigma$ is the conductivity of copper and $H$ is the external magnetic field.

The current at both edges under skin effect is approximated as

$$
J_{\text {skin-edge }}=\frac{I}{2 t \delta}
$$

where $t$ is the thickness of copper trace and $\delta$ is the skin depth. Finally the current density on both edges is the result of two effects

$$
\begin{aligned}
J_{\text {edge-left }} & =J_{\text {skin-edge }}+J_{\text {prox-edge }} \\
J_{\text {edge-right }} & =J_{\text {skin-edge }}-J_{\text {prox-edge }}
\end{aligned}
$$

Under the filament approximation, the summation of the left edge current $I_{l}$ and right edge current $I_{r}$ is the entire current $I$. Current distribution is supposed to be

$$
\begin{aligned}
I_{l} & =I \frac{J_{\text {edge-left }}}{J_{\text {edge-left }}+J_{\text {edge-right }}} \\
I_{r} & =I \frac{J_{\text {edge }- \text { right }}}{J_{\text {edge-left }}+J_{\text {edge-right }}}
\end{aligned}
$$

Finally, the magnetic field is solved with (2) and (8).

Comparing to the original filament method, the proposed method considers two current loops at edges instead of one current loop in middle of the conductor trace. Besides, the proposed method considers the redistribution of magnetic field with the knowledge of current density distribution. The accuracy of the proposed approach is validated with randomly selected coil geometry against FEM simulations. In Fig. 3, the result of a spiral coil of 6 turns with $w=5 \mathrm{~mm}, t=$ $0.07 \mathrm{~mm}, R_{\text {out }}=70 \mathrm{~mm}$ is shown. The real magnetic field distribution is not linear. Comparing to the result with the filament method from [24], the result of proposed method better presents the mean value of the real distribution. 


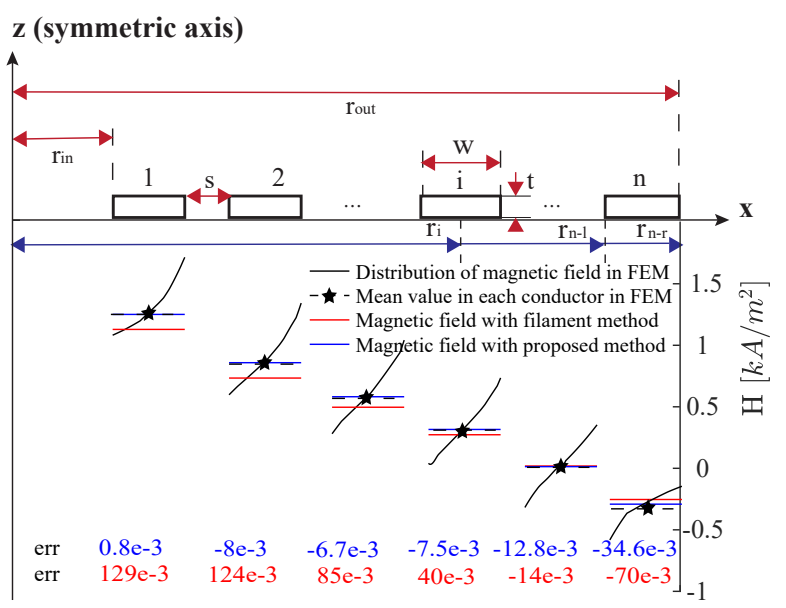

Fig. 3. Concentric circular conductors with rectangular cross sections and magnetic filed distribution with a current excitation of $1 \mathrm{~A}$ at $500 \mathrm{kHz}$ for 6-turn coil with $w=5 \mathrm{~mm}, t=0.07 \mathrm{~mm}, R_{\text {out }}=70 \mathrm{~mm}$. The errors are absolute errors: err $=$ modeling result - mean value of simulation result.

\section{B. Modeling of self inductance}

The current sheet method is commonly used for modeling the inductance, which considers multiple spiral turns as one single current sheet [17]. However, the error increases when the ratio between $s$ and $w$ is bigger than 3 . The data-fitting technique for a predefined monomial model is also an option [17], but the existing model is only for polygonal spiral coils, not for circular coils.

In this section, the spiral coil is modeled as coaxial circular conductors. The inductance of the circular spiral coil can be expressed as the sum of self inductance of each circular sheet and mutual inductance between each pair of sheets. This method intends to avoid error caused by large space between turns.

$$
L_{\text {spiral }}=\sum_{i} L_{i}+\sum_{i, j} M_{i, j}, \forall i, j \in n
$$

This summation method works for all the $\mathrm{s}$ and $\mathrm{w}$ ranges. The self inductance and mutual inductance in (9) are [15][27]

$$
\begin{aligned}
L_{i}= & \mu r_{i}\left[\ln \left(\frac{2 r_{i}}{w}\right)+0.9+0.05 \frac{w^{2}}{r_{i}^{2}}\right] \\
M_{i, j}= & \frac{\left.\mu\left(r_{i}+r_{j}\right)\right)}{2}\left[\ln \left(\frac{r_{i}+r_{j}}{w+s}\right)-0.6+0.7\left(\frac{w+s}{r_{i}+r_{j}}\right)^{2}\right] \\
& +\left(0.2+\frac{\left(r_{i}+r_{j}\right)^{2}}{12(w+s)^{2}}\right) \frac{\mu \omega^{2}}{2\left(r_{i}+r_{j}\right)}
\end{aligned}
$$

Again, the analytic model of PCB coil inductance are checked against the FEM simulations for randomly selected coil geometries. Fig. 4 shows the inductances extracted from ANSYS Maxwell 3D and analytic modeling for the coils with 6 turns and outer radius of $75 \mathrm{~mm}\left(R_{\text {out }}=75 \mathrm{~mm}\right)$. With the constraints of outer radius and number of turns, the maximum trace width is $9 \mathrm{~mm}$. Trace width and space between turns are swept to generate more designs. Due to the limit in According to Fig. 4, the analytic result correlates well with FEM result for all considered coil geometries.

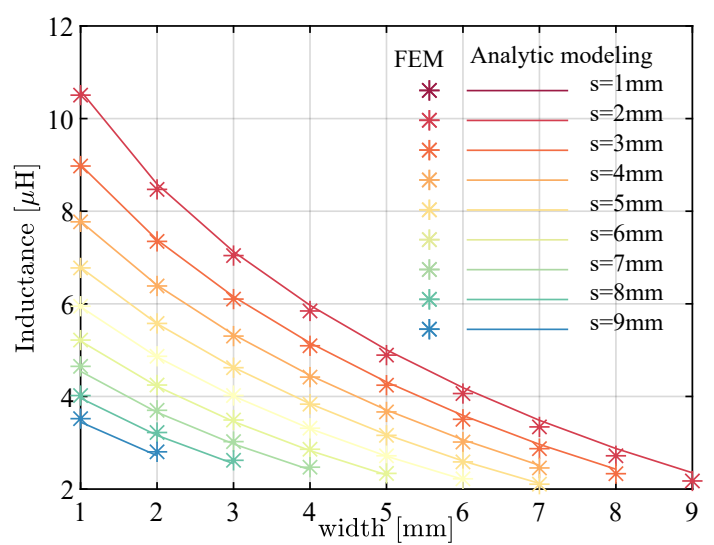

Fig. 4. Inductance extracted from FEM simulation and from analytic modeling. The studied coil has 6 turns and outer radius of $75 \mathrm{~mm}$. The trace width and space between turns vary with a step of $1 \mathrm{~mm}$.

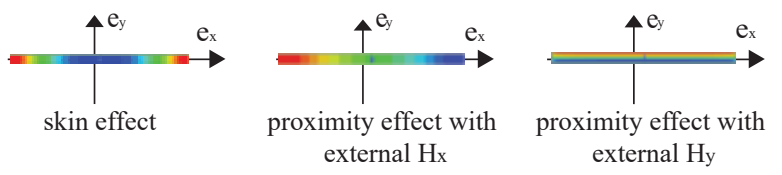

Fig. 5. Current density distribution caused by skin effect, proximity effect in a conductor track with rectangular cross section and infinite long in $e_{z}$ direction.

\section{Modeling of AC resistance}

Unlike traditional inductor design, there is no empirical model calculating resistance of PCB spiral coils. Besides, unlike circular cross-section conductor [28], there is no closedform analytic expression to calculate the resistance of a rectangular cross-section coil. Therefore, a hybrid FEM database and magnetic field calculation method is proposed in order to increase the accuracy in resistance calculation.

1) Orthogonality of conduction and proximity losses: In the rectangular cross-sectional conductors, the independence of conduction and proximity losses remains as in the round wires. Firstly, the superposition of current densities due to skin effect and proximity effect is involved.

The total loss $P_{a c}$ for unit length can be calculated as follows:

$$
\begin{aligned}
P_{a c}= & \frac{1}{2 \sigma} \int_{S}\left(J_{\text {cond }}+J_{\text {prox }, x}+J_{\text {prox }, y}\right) \\
& \times\left(J_{\text {cond }}+J_{\text {prox }, x}+J_{\text {prox }, y}\right)^{*} d S .
\end{aligned}
$$

In the above equation, all the cross-term products are zero. Therefore (11) can be simplified as the superposition of conduction losses (caused by the influence inside the conductor including skin effect loss under high frequency excitation) and proximity loss (caused by the influence of other conductors) .

$P_{a c}=\frac{1}{2 \sigma} \int_{S}\left(J_{\text {cond }} J_{\text {cond }}^{*}+J_{\text {prox }, x} J_{\text {prox }, x}^{*}+J_{\text {prox }, y} J_{\text {prox }, y}^{*}\right) d S$

2) FEM database to evaluate resistance: In rectangular cross-sectional conductors, expressions to evaluate both types 


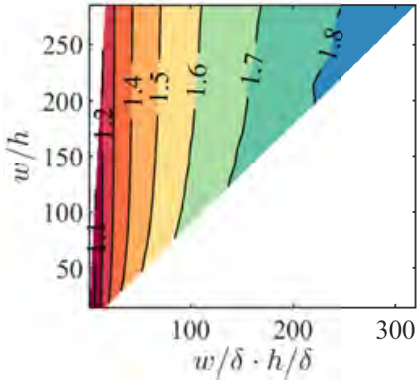

(a)

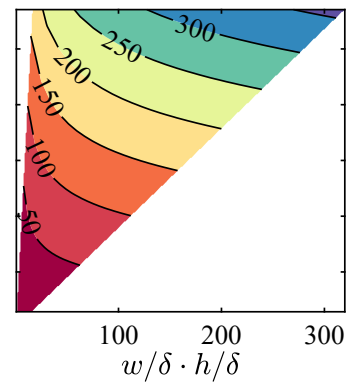

(b)
Fig. 6. Dimensionless functions extracted from FEM simulation (a) $\Phi_{\text {cond }}$ (b) $\Phi_{\text {prox }} . \Phi_{\text {cond }}$ is obtained by setting a current excitation through a straight conductor. Balloon boundary is setted at the region edges. $\Phi_{\text {prox }}$ is obtained by setting a constant magnetic field in $e_{y}$ direction. Variant magnetic vector potential is set at the simulation region edges.

of losses are given in [18]:

$$
\begin{aligned}
R_{\text {condu.l. }} & =\frac{1}{w h \sigma} \Phi_{\text {condrec }}(w / \delta, h / \delta) \\
R_{\text {proxu.l.x }} & =\frac{4 \pi}{\sigma} \Phi_{\text {proxrec }}(w / \delta, h / \delta) \hat{H}_{1, x}^{2} \\
R_{\text {proxu.l.y }} & =\frac{4 \pi}{\sigma} \Phi_{\text {proxrec }}(w / \delta, h / \delta) \hat{H}_{1, y}^{2}
\end{aligned}
$$

where $H_{1, x}$ and $H_{1, y}$ are the magnetic field generated by 1 A current and are calculated with magnetic field model in Section III A. $\Phi$ are dimensionless functions which describe frequency and geometry dependency of resistance. With current $I$ through the spiral coil, the power loss is:

$$
P_{a c, u . l .}=\frac{1}{2}\left(R_{\text {condu.l. }}+R_{\text {proxu.l.x }}+R_{\text {proxu.l.y }}\right) I^{2}
$$

Since $\Phi_{\text {cond }}$ and $\Phi_{\text {prox }}$ in (13) don't have closed-form analytic expressions, FEM is used to calculate the value of these functions. In ANSYS Maxwell 2D, the conductor is modeled as a straight conductor with infinite length. The geometry and frequency are swept according to TABLE II. In addition, copper thickness $t$ is swept from $0.035 \mathrm{~mm}$ to $0.21 \mathrm{~mm}$.

It has to be noted that FEM is only used to generate database for $\Phi$ in equation (13) rather than directly for AC resistance because $\Phi$ has fewer free dimensions and requires a much smaller database.

In Fig. 6a and Fig. 6b, the $\mathrm{x}$-axis $w / \delta \cdot h / \delta$ is the crosssection area of the conductor with respect to the effective conducting area. The value smaller than 1 means the frequency effect can be ignored. The bigger value means the stronger influence of the high frequency on the effective conducting area. The $\mathbf{y}$-axis $w / h$ represents the form factor of the crosssection, with the value close to 1 , the cross-section is close to a square area. The function model can be obtained by importing these data into Matlab and using the curve fitting tool. In the curve fitting tool, the $\mathrm{x}, \mathrm{y}, \mathrm{z}$ data in Fig. 6 are taken as inputs to train the model. The output of the curve fitting tool is dimensionless function $\Phi$. In this manuscript, our function is obtained with the Biharmonic interpolation method.

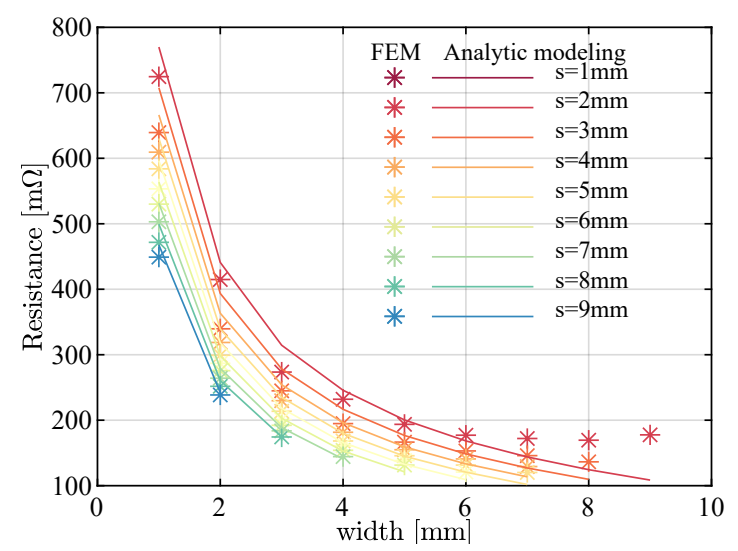

Fig. 7. Resistance at $500 \mathrm{kHz}$ extracted directly from FEM simulation and from analytic calculation in (13). The studied coil has 6 turns and outer radius of $75 \mathrm{~mm}$. The trace width and space between turns vary with a step of 1 $\mathrm{mm}$.

3) FEM Verification: The modeling of resistance of spiral coil is verified with the same case for self inductance verification. As shown in Fig. 7, the modeling matches well with the FEM results in most cases. With the increase of the trace width $(w>8 \mathrm{~mm})$, the error becomes larger, which results from the simplification while modeling the magnetic field as well as the simplification by using concentric circles instead of spiral coils. However, considering that in reality, very wide PCB traces are rarely used in spiral coils, the proposed fast modeling of resistances is still applicable in most cases.

\section{Modeling of mutual inductance}

Power transfer requires at least two coils. As shown in Fig. 8, in this paper, two coils are placed face to face on primary side and secondary side respectively with air between them. In MV application, the smallest distance $d$ between them is determined by the insulation voltage taking into account type of insulation, overvoltage category and pollution degree. Mutual inductance is calculated based on method in [29], where the planar spiral coils are simplified to co-central loops and mutual inductance $M$ is calculated as the summation of mutual inductance between each circular loops.

$$
M=\sum_{i=1}^{M} \sum_{j=1}^{N} M_{i, j}\left(r_{i}, r_{j}, d\right)
$$

As shown in Fig. 8, $i$ and $j$ are the respectively $i^{\text {th }}$ and $j^{\text {th }}$ turn in primary and secondary coils.

Two coils are fabricated and taken under test with a different air gap between the two coils. The test result, the calculation result, and FEM results are shown in Fig.9. The three curves superposed each other well. The coupling factor shows a clear decline with a longer distance.

\section{DESIGN PROCESS}

In this section, the performance of various coil geometries within the design space in Table II are evaluated based on the aforementioned mathematical model. The design procedure 


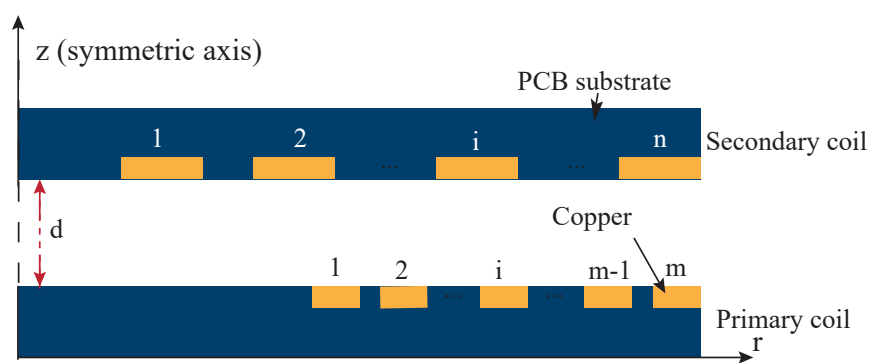

Fig. 8. A set of IPT PCB coils in z plane. Copper trace on the outer layer of PCB is the focus of this paper and PCB substrate design is not discussed here. The air gap between primary coil and secondary coil is $d$. For illustration purpose, primary coil has $m$ turns and secondary coil has $n$ turns.

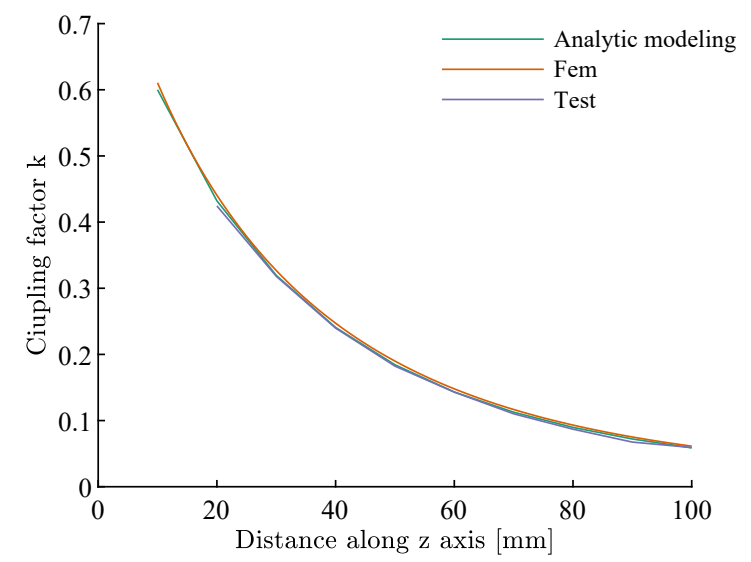

Fig. 9. Coupling between two coils: primary coil has 6 turns, turns width of $5.2 \mathrm{~mm}$, turns gap of $2 \mathrm{~mm}$, outer radius of $70 \mathrm{~mm}$; secondary coil has 7 turns, turns width of $2 \mathrm{~mm}$, turns gap of $1 \mathrm{~mm}$ and outer radius of $70 \mathrm{~mm}$.

shown in Fig. 10 is based on brute-force search in the entire design space. Before the start of design approach, the distance between primary and secondary coils in IPT system should be defined and this is addressed in detail thereafter in subsection A. In the design procedure, firstly, individual coils are characterized with the inductance model and resistance model proposed in Section III. Secondly, coils are grouped into pairs, considering all the combinations. Mutual inductance of each pair is calculated with the analytic model in Section III and based on the coil distance. Thirdly, the power transfer capability of each coil pair is evaluated as illustrated thereafter in subsection B. Finally, designs are filtered by the constraints illustrated in subsection $\mathrm{C}$.

Various coils within the design space in TABLE II are evaluated based on the analytic modeling. Width of copper trace is swept from $1 \mathrm{~mm}$ to $20 \mathrm{~mm}$, the space between spiral turns is swept from $1 \mathrm{~mm}$ to $20 \mathrm{~mm}$ and the outer radius of circular coil is swept from $10 \mathrm{~mm}$ to $75 \mathrm{~mm}$. The minimum space of $1 \mathrm{~mm}$ is taken to avoid electric breakdown between coil turns according to IPC9592 standard. For these ranges, 10 equal steps are used in between. The number of turns is from 1 to the maximum allowable turns with defined $w, r_{\text {out }}, s$. Each set of these variables characterize uniquely each single coil. In one coil pair, the primary and secondary don't need to be identical. In total, 1426 single coils with different geometries

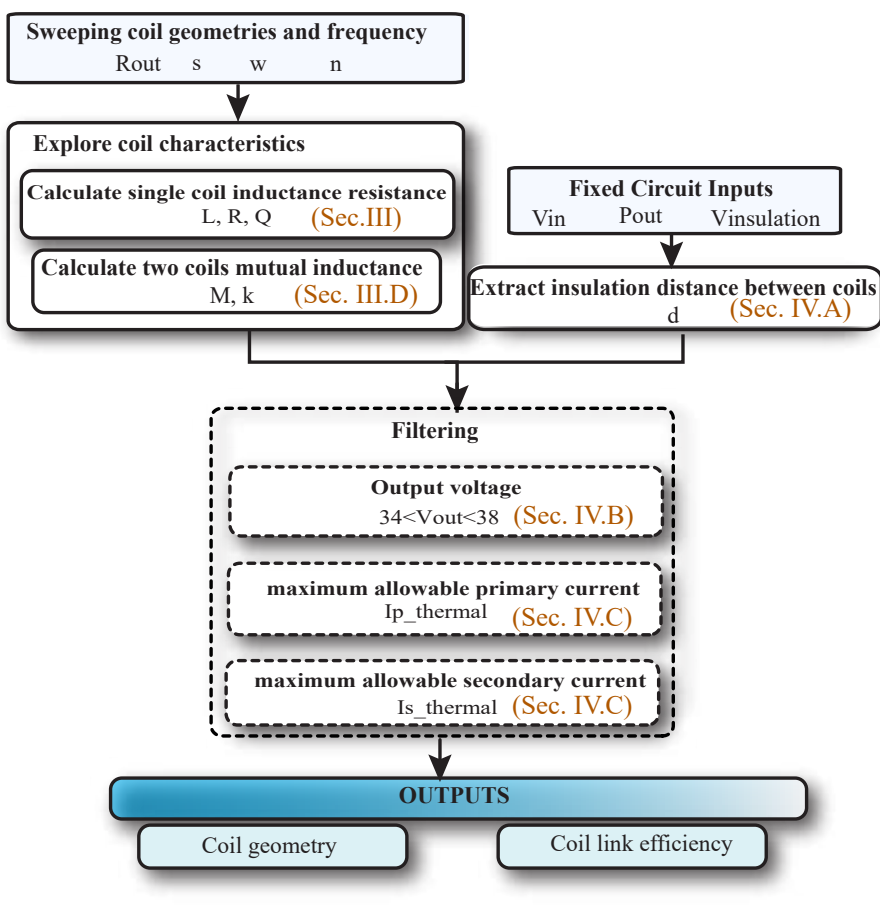

Fig. 10. Design procedure followed in this paper to optimize coil pairs in IPT system. The output of this procedure is all the possible primary and secondary coil geometries which meet application requirements. The variable notations in this flow chart correspond to those in Section II. For each design step, the corresponding paper section is listed.

are designed and $1426^{2}$ combination of coil pairs are explored considering 11 different operating frequencies. With 4 Intel i7 cores, 16GB RAM, it took 24 hours to finish the calculation of all the coils. The inductance and resistance of coils are obtained by modeling, namely, $L_{p}, L_{s}, R_{p}, R_{s}, M$. The figure of merits coupling factor and quality factor are defined as $k=\frac{M}{\left.\sqrt{(} L_{p} L_{s}\right)}, Q_{p}=\frac{\omega L_{p}}{R_{p}}, Q_{s}=\frac{\omega L_{s}}{R_{s}}$. Furthermore, the current through coils, output voltage over the secondary coil, and coil link efficiency are calculated.

\section{A. Insulation distance}

Insulation distance is determined by interpolation from Table 8 and Table 9 in standard IEC 61800-5-1 [22]. The clearance distance for basic insulation between two coils is defined according to the working voltage, the impulse voltage, the temporary overvoltage and the recurring peak value of working voltage. As shown in TABLE III, the r.m.s. value of working voltage is $6 \mathrm{kV}$. The impulse voltage and temporary overvoltage are determined by this working voltage. The impulse voltage also depends on the overvoltage category. With the overvoltage category II, the impulse voltage is $24.8 \mathrm{kV}$, and temporary overvoltage is $24 \mathrm{kV}$. The final clearance distance takes the maximum value among the three distances. However, in practical design considering the complex environment, an extra safety margin is always recommended. Finally, $60 \mathrm{~mm}$ is taken as the distance between two coils to realize basic insulation for the $6 \mathrm{kV}$ system. It should be noted that the standard used to define insulation distance should be selected based on different applications. 


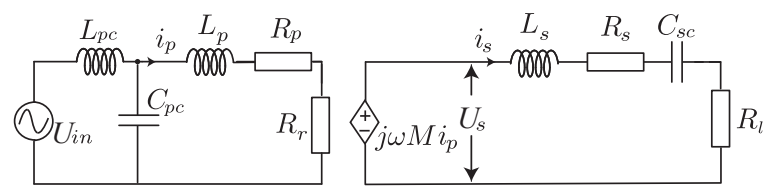

Fig. 11. Equivalent model of IPT system with LCL-S compensation network.

\section{B. Outputs of coil links}

In this section, AC voltages and currents are all in r.m.s values, and the subscript $p$ refers to primary side, while $s$ indicates secondary side.

Equivalent model of coil link is shown in Fig. 11. The coil link efficiency is the product of the efficiency of primary side and secondary side:

$$
\eta_{\text {coillink }}=\eta_{p} \eta_{s}=\frac{R_{r}}{R_{r}+R_{p}} \frac{R_{l}}{R_{l}+R_{s}}
$$

where, $R_{r}=\frac{\omega^{2} M^{2}}{R_{l}+R_{s}}$ is the reflected resistance of secondary on the primary side. $R_{l}$ is the equivalent load. Provided ideal compensation [21], $R_{l}$ is obtained by solving the equation of output power

$$
\frac{U_{s}^{2}}{\left(R_{s}+R_{l}\right)^{2}} R_{l}=P_{\text {out }}
$$

where $R_{s}$ is the resistance of secondary coil. By solving (17), the load resistance is

$$
R_{l}=\left(\frac{U_{s}^{2}}{P_{\text {out }}}-2 R_{s}\right)+\frac{\sqrt{\left(\frac{U_{s}^{2}}{P_{\text {out }}}-2 R_{s}\right)^{2}-4 R_{s}^{2}}}{2}
$$

Finally, the DC output voltage after half bridge rectifier is obtained

$$
U_{d c}=\frac{\pi}{\sqrt{2}} \frac{U_{s} R_{\text {load }}}{R_{\text {load }}+R_{s}}
$$

\section{Design constraints}

By pairing all possible coil pairs, different relations are generated between input and output voltage. The output voltage for each coil pair is shown in Fig.12, with many combinations producing output voltage outside the desired range. Useful coil pairs need to be extracted for the analysis. With the current and voltage outputs obtained in the previous section, the coil designs are filtered by two criteria, namely the output voltage range and heat dissipation limit.

The output voltage at the rated power is limited in the range $34 \mathrm{~V}-38 \mathrm{~V}$. The coil pairs that violate this voltage range are filtered out and not analysed further.

Regarding heat dissipation, natural convection is considered in this work. With natural cooling, thermal resistances are shown in TABLE IV. Since $\theta_{C u} \ll \theta_{S A}$, the thermal resistance

TABLE III: Minimum clearance distance required to provide basic insulation

\begin{tabular}{||lclc||}
\hline System working voltage & $6 \mathrm{kV}$ & $\begin{array}{l}\text { Minimum } \\
\text { clearance distance }\end{array}$ & $28.4 \mathrm{~mm}$ \\
\hline $\begin{array}{l}\text { Impulse voltage of over- } \\
\text { voltage category II }\end{array}$ & $24.8 \mathrm{kV}$ & $\begin{array}{l}\text { Minimum } \\
\text { clearance distance }\end{array}$ & $32.8 \mathrm{~mm}$ \\
\hline Temporary overvoltage & $24 \mathrm{kV}$ & $\begin{array}{l}\text { Minimum } \\
\text { clearance distance }\end{array}$ & $54.84 \mathrm{~mm}$ \\
\hline
\end{tabular}

of primary and secondary coil are dominated by the copper coverage surface $\pi\left(R_{\text {out }}^{2}-R_{\text {in }}^{2}\right)$ and copper thermal resistance $\theta_{S A}$. Since the thickness of FR4 is minimal $(\approx 1.55 \mathrm{~mm})$, the heat is approximately dissipated equally through both sides of the PCB board, thus

$$
\begin{aligned}
R_{\text {th }} & =R_{\text {th-uppersurface }}+R_{\text {th-lowersurface }} \\
& =\frac{\theta_{S A}}{\pi\left(R_{\text {out }}^{2}-R_{\text {in }}^{2}\right)} \frac{1}{1+\frac{1000}{1067}} \frac{w}{w+s}
\end{aligned}
$$

The current limited by the heat dissipation capability is

$$
I_{\text {cal }}=\sqrt{\frac{\triangle T}{R_{t h} R_{\text {coil }}}}
$$

where $R_{\text {coil }}$ is the resistance of coil obtained from analytic modeling; $\triangle T$ is the allowed temperature rise.

Another current restriction comes from standard IPC-2221 [30], where the same current limit are used under variant current excitation frequency. By curve fitting, the maximum current in the external copper layer is:

$$
I_{\text {std }}=(\text { width } \cdot \text { thickness })^{0.725} 0.048 \triangle T^{0.44}
$$

The current limited by thermal dissipation capability takes the minimum value between the $I_{s t d}$ and $I_{c a l}$. The comparison of $I_{s t d}$ and $I_{c a l}$ is shown in Fig. 13. $I_{s t d}$ extracted from the standard is usually higher than the calculated value $I_{c a l}$.

\section{RESULTS OF OPTIMIZATION TOOL}

In the entire design space in TABLE II, $1426^{2} \times 11$ coil pair designs were explored.

\section{A. Influence of Coil geometry}

High coupling factor $k$ and high quality factor $Q_{p}, Q_{s}$ are the figure of merits to achieve high coil link efficiency. Therefore, they are considered as main performance indicators for coils. In this section, several important coil geometry properties are explored. Their influence on $k, Q$ and coil link efficiency are analyzed. The results shown in this section are all at an operating frequency of $500 \mathrm{kHz}$.

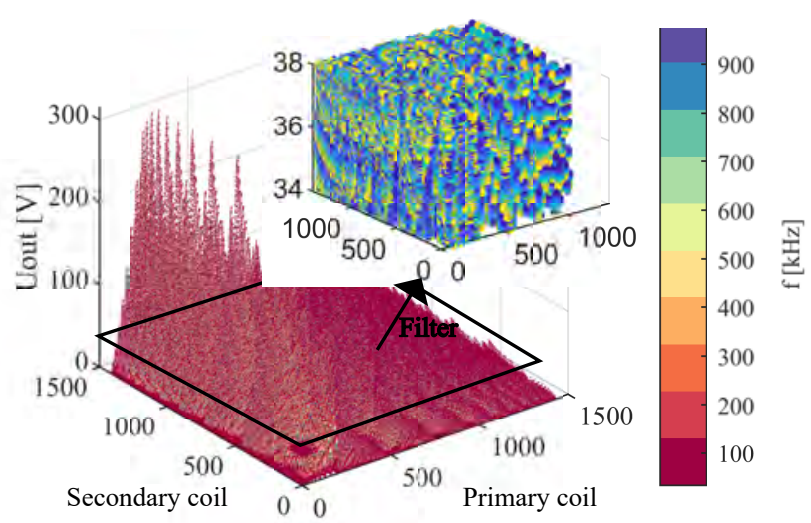

Fig. 12. Output voltages of all the coil combinations. In total, $1426^{2} \times 11$ designs are shown. After filtering by design constraints, 18868 designs are left and are shown in the zoom-in view. 


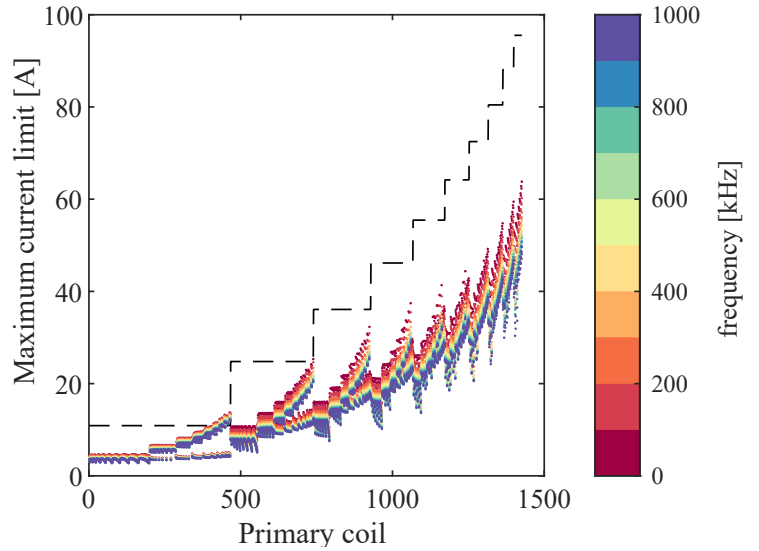

Fig. 13. Two different current limitations: black dashed represent the current limitation extracted from standard IPC-2221 [30]; colorful dots represent current limit calculated by conductor loss. Four parameters are swept from inner loop to outer loop following the order: $n, r_{\text {out }}, s, w$. Four parameters increase as the coil numbering rises.

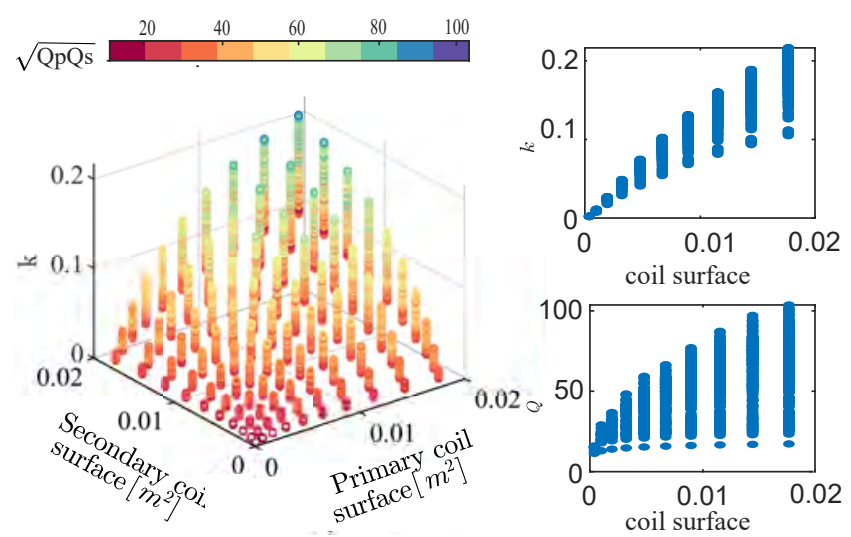

Fig. 14. The relation between the coil surface area, coupling factor and quality factor. The $3 \mathrm{D}$ view is on left. On the right, $2 \mathrm{D}$ plots are shown with identical primary and secondary coils.

1) Surface area of coil: The surface area here is defined only by coils' outer radius and omits the differences of inner radius since outer radius defines final size of the coil. The surface area dominates the coupling factors between two coils and the quality factor of a single coil. As shown in Fig. 14, when the surface area increases, the maximum reachable $k$ and $Q$ also increase. A large outer radius also brings high achievable efficiency.

2) Inner radius of coil: To minimize eddy current effects and get higher coupling, majority of previous studies recom-

TABLE IV: Thermal resistance of PCB coil

\begin{tabular}{||ccl||}
\hline Name & Value & Description \\
\hline$\theta_{\mathrm{Cu}}$ & $0.25 \frac{{ }^{\circ} \mathrm{Ccm}}{W}$ & $\begin{array}{l}\text { Thermal resistance of } 1 \mathrm{~cm}^{2} \\
\text { copper }\end{array}$ \\
\hline$\theta_{F R 4}$ & $67.43 \frac{{ }^{\circ} \mathrm{Ccm}^{2}}{W}$ & $\begin{array}{l}\text { Thermal resistance of FR4 } \\
\text { substrate in } 1 \mathrm{~cm}^{2} \text { and thick- } \\
\text { ness of } 0.155 \mathrm{~cm}\end{array}$ \\
\hline$\theta_{S A}$ & $1000 \frac{{ }^{\circ} \mathrm{Ccm}^{2}}{W}$ & $\begin{array}{l}\text { Thermal resistance from } 1 \mathrm{~cm}^{2} \\
\text { PCB surface to air }\end{array}$ \\
\hline
\end{tabular}
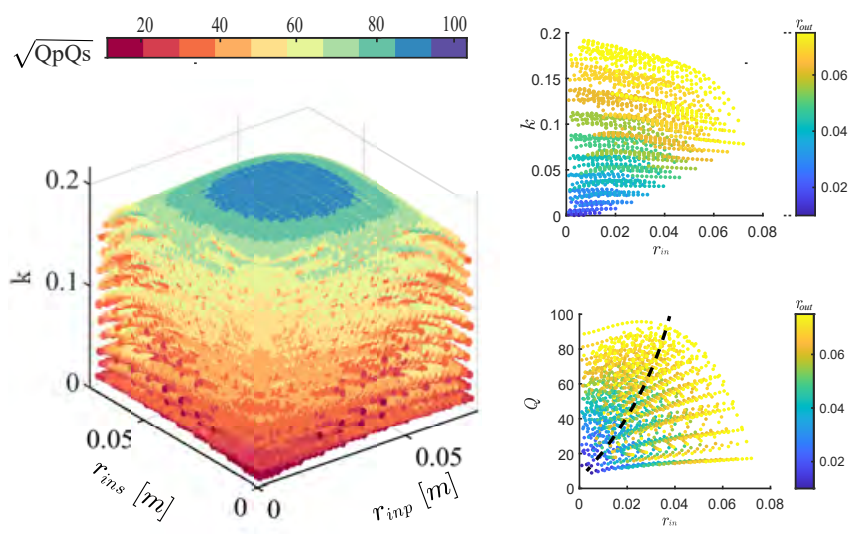

Fig. 15. The relation between the coil inner radius, coupling factor and quality factor. The 3D view is on left. On the right, 2D plots are shown with identical primary and secondary coils.

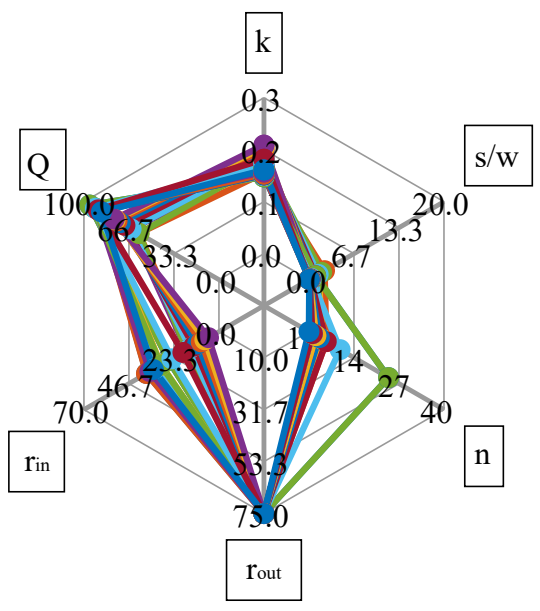

Fig. 16. Radar plot for designs in $500 \mathrm{kHz}$ with two optimal goal $k, Q$ as well as interesting parameters in each leg. Each color represents one design.

mend to eliminate the most inner turns by leaving a hole in the middle of the spiral coil [25], [31]. The dependence on coil inner radius of $\mathrm{k}, \mathrm{Q}$ are presented in Fig. 15. The 3D plot includes the consideration of combinations of different primary and secondary coils. On the other hand, the 2D plot considers only the combination of identical primary and secondary coils. From the 2D plots, it is apparent that $\mathrm{Q}$ is expected to drop sharply after the peak. With the decrease in different outer radius, the optimal inner radius for peak $\mathrm{Q}$ is decreasing. For the ten outer radius $[10,17.2,24.4,31.7,38.9,46.1,53.3,60.6$, $67.875]$, the corresponding inner radius to achieve highest Q are $[5,8.2,11.4,14.6,17.9,21.1,24.3,27.6,30.8,36]$. The units are in millimeters.

3) Space-width ratio: The space between each turn affects $Q$. A narrow space brings higher inductance, but it also means a stronger proximity effect leading to higher coil resistance. Therefore an optimal range exists to achieve a maximum $Q$. Based on our database, $\frac{s}{w} \leq 1.5$ is a reasonable optimal range.

4) Number of turns and trace width of coil: Based on our data set, the optimal $w$ to achieve $Q$ and $k$ are scattered over 


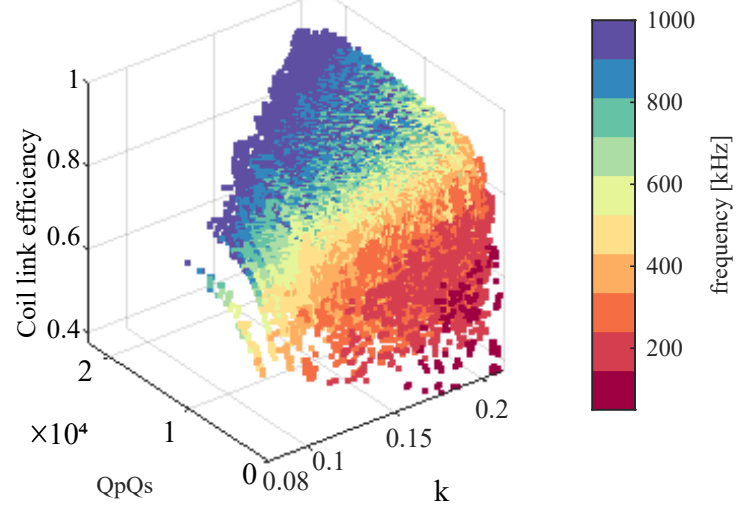

Fig. 17. The relation between frequency, coupling factor $\mathrm{k}$, quality factor $Q_{p} Q_{s}$ and coil link efficiency. Higher frequency leads to higher $\mathrm{Q}$ in the defined frequency range. The designs which have small $\mathrm{k}$ show stronger limit on achievable maximum Q. Higher $\mathrm{k}$ and $\mathrm{Q}$ lead to higher efficiency.

the entire design space. For the turns number $n$, there is no general optimal range to achieve maximum $k$. On the other hand, coil composed of a single turn $(n=1)$ has prominent high $Q$.

5) Conclusion: In order to see the optimal design range of each geometry parameter, a radar plot is shown in Fig.16, where each leg of the radar is a different design parameter. The design points on each leg defines the optimal range for this parameter. For example, the outer radius are all at $75 \mathrm{~mm}$, the $\frac{s}{w}$ ratio is less than 2 , inner radius and turns number are scattered in a larger range in the design space and no optimal design range is observed. This shows that the larger outer radius, the higher performance. And $\frac{s}{w}$ is a delicate parameter which has a upper limit of optimal design range.

\section{B. Influence of operating frequency}

The results discussed in this section consider only the filtered designs. In Fig. 17, there is a clear trend that in the defined frequency range, quality factor increases as frequency increases, which is explained as the increase of coil resistance caused by high frequency is not comparable to the increase of inductive reactance.

In Fig. 18, although the maximum attainable coil link efficiency increases as the frequency keeps increasing, 500 $\mathrm{kHz}$ is chosen over $1 \mathrm{MHz}$ for the experimental demonstration thereafter. Because switching at $500 \mathrm{kHz}$ offers a wide choice for semiconductors, silicon devices as well as wide band-gap devices are all usable in this frequency range.

\section{Pareto plot}

As shown in Fig. 19, trade-off between high coil link efficiency and power density exists in the IPT coil design. The coil link efficiency is from the primary coil to the secondary coil and exclude the compensation network and converters. The power density is defined as $\frac{2 P_{\text {out }}}{\left(S_{p}+S_{s}\right) d}$, where $P_{\text {out }}$ is the output power, $S_{p}$ and $S_{s}$ are the surface area of primary and secondary coils and $d$ is the distance between two coils. The Pareto front for each frequency is clearly visible.

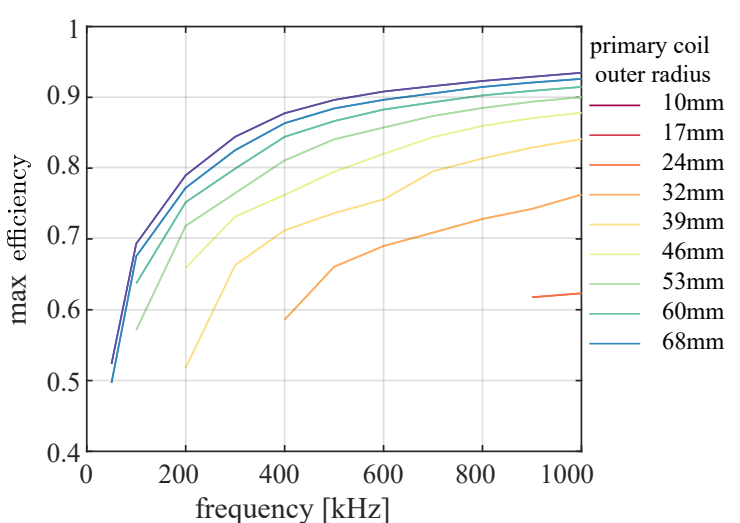

Fig. 18. Maximum achievable coil link efficiency under different operating frequencies and different outer radius within the design space.

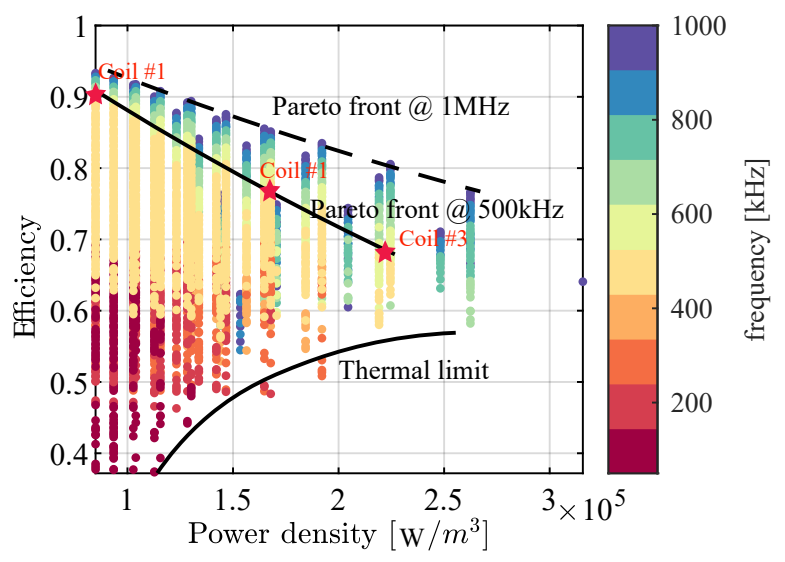

Fig. 19. Pareto plot of coil link efficiency and power density in the full range of design space. Three designs under $500 \mathrm{kHz}$ operating frequency are selected for prototyping and marked as star in the figure.

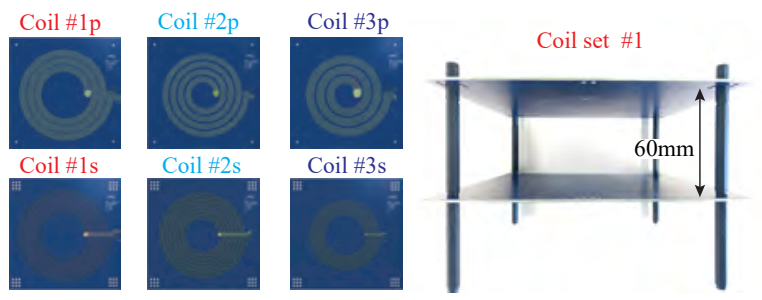

Fig. 20. Top view of 6 prototype coils and front view of coil set 1 with primary coil on bottom and secondary coil on top. Spaces between two coils are controlled by the length of spacers.

\section{EXPERIMENTAL VERIFICATION}

In Fig. 19, three designs on the Pareto front of $500 \mathrm{kHz}$ are selected to prototype and the geometries of three coil sets are shown in TABLE V. Designs are selected to verify different metrics: coil 1 has the highest efficiency, coil 3 has the highest power density and coil 2 takes the trade-off between efficiency and power density into consideration. The prototype of coil pairs are shown in Fig. 20.

1) Modeling verification: The inductance and resistance modeling is verified with test using BK 895 LCR meter which has $2 \%$ accuracy. The test is executed at $500 \mathrm{kHz}$. The resistance and inductance value obtained from both analytic modeling and test are shown in TABLE VI. The analytic 
TABLE V: Parameters of the prototyped coils

\begin{tabular}{||cccccc||}
\hline Specifications & Characteristics & Prototype I & Prototype II & Prototype III & units \\
\hline \hline primary coil outer radius & $r_{\text {outp }}$ & 75 & 68.5 & 68.5 & $\mathrm{~mm}$ \\
\hline primary coil trace width & $w_{p}$ & 10.5 & 8.6 & 12.4 & $\mathrm{~mm}$ \\
\hline primary coil turns gap & $s_{p}$ & 1 & 2.9 & 2.9 & $\mathrm{~mm}$ \\
\hline primary coil turns number & $n_{p}$ & 3 & 4 & 3 & - \\
\hline primary coil space-width ratio & $\frac{s_{p}}{w_{p}}$ & 0.1 & 0.33 & 0.23 & - \\
\hline primary coil quality factor & $Q_{p}$ & 70.15 & 61.75 & 61.75 & - \\
\hline secondary coil outer radius & $r_{\text {outs }}$ & 75 & 68.5 & 55.5 & $\mathrm{~mm}$ \\
\hline secondary coil trace width & $w_{s}$ & 6.7 & 4.8 & 2.9 & $\mathrm{~mm}$ \\
\hline secondary coil turns gap & $s_{s}$ & 1 & 1 & 1 & $\mathrm{~mm}$ \\
\hline secondary coil turns number & $n_{s}$ & 5 & 7 & 6 & - \\
\hline secondary coil space-width ratio & $\frac{s_{s}}{w_{s}}$ & 0.14 & 0.2 & 0.34 & - \\
\hline secondary coil quality factor & $Q_{s}$ & 70.7 & 65.8 & 56.1 & - \\
\hline
\end{tabular}

TABLE VI: Modeling and test result of inductance and resistance of 3 coil pairs

\begin{tabular}{|c|c|c|c|c|c|c|}
\hline \multicolumn{2}{|c|}{ Coil pair } & $L_{p}[\mu H]$ & $L_{s}[\mu H]$ & $R_{p}[m \Omega]$ & $R_{s}[m \Omega]$ & $M[\mu H]$ \\
\hline \multirow{4}{*}{1} & Ana & 1.117 & 3.017 & 50 & 134 & 0.279 \\
\cline { 2 - 7 } & Test & 1.12 & 3.02 & 48 & 126 & 0.274 \\
\cline { 2 - 7 } & Error & $<1 \%$ & $<1 \%$ & $3.6 \%$ & $6.5 \%$ & $<1 \%$ \\
\hline \multirow{4}{*}{2} & Ana & 1.18 & 4.736 & 60 & 226 & 0.302 \\
\cline { 2 - 7 } & Test & 1.24 & 4.74 & 60 & 240 & 0.3 \\
\cline { 2 - 7 } & Error & $5.3 \%$ & $<1 \%$ & $<1 \%$ & $4.2 \%$ & $<1 \%$ \\
\hline \multirow{3}{*}{3} & Ana & 0.59 & 4.129 & 30 & 231 & 0.165 \\
\cline { 2 - 7 } & Test & 0.628 & 4.13 & 42 & 195 & 0.16 \\
\cline { 2 - 7 } & Error & $6.5 \%$ & $<1 \%$ & $26 \%$ & $18 \%$ & $<1 \%$ \\
\hline
\end{tabular}

model of self and mutual inductance shows good match with the test results. Large error around 5\% appears at large PCB trace width. The largest error of resistance $26 \%$ appears at large trace width. This is due to the drop of resistance model accuracy for large trace width. If looking into the absolute error, $26 \%$ of coil resistance corresponds to $12 \mathrm{~m} \Omega$ which is small comparing to parasitic resistance in peripheral circuit.

The test of mutual inductance under different distances are also performed and results are shown in Fig. 21. The analytic model of mutual inductance shows high accuracy. Moreover, in order to explore the influence of lateral displacement between two coils which could happen due to wrong installation or vibrations, the test with a lateral displacement of $5 \mathrm{~mm}$ in each direction are executed. The misalignment in the direction $x y$ induces the largest discrepancy and its results are shown in Fig. 21. It can be seen that small lateral displacements have negligible influence on mutual inductance.

\section{A. Power test}

The first coil pair detailed in TABLE V is tested with testbench shown in Fig. 22. The coil set is tested with an air gap of $60 \mathrm{~mm}$, nominal load of $10 \Omega$, input voltage of $80 \mathrm{~V}$ and operating frequency of $500 \mathrm{kHz}$.

Other components in the test bench are as follows. The transmitter coil is connected to a GaN HEMTs based full bridge inverter (Infineon, EVAL-1EDF-G1-HB-GAN). The receiver coil is connected to Schottky diode based passive half bridge rectifier (ST, STPS30M60DJF). Tektronix TCPA300

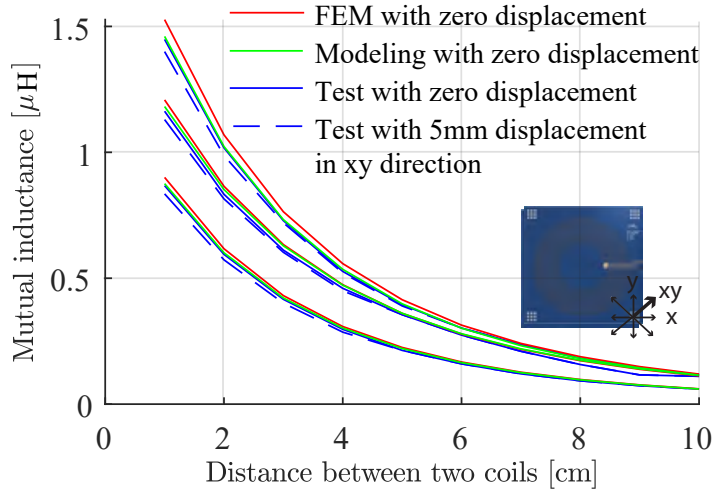

Fig. 21. Mutual inductance for three different coil sets under differen transfer air gaps with zero or $5 \mathrm{~mm}$ lateral displacement in $x y$ direction. Along with the increase of distance between primary and secondary coil, the mutual inductance drops quickly. Test result, FEM result and modeling result correlate well with each other.

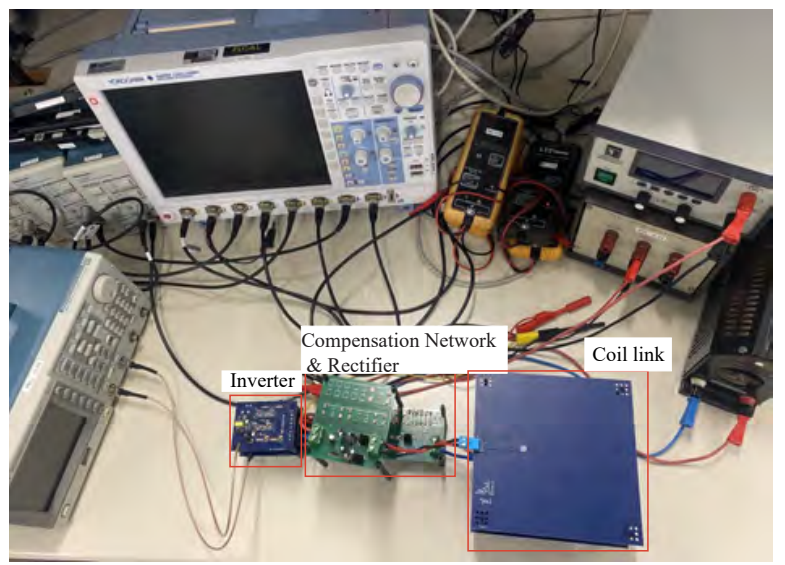

Fig. 22. Test set-up of inductive power transfer from $80 \mathrm{~V}$ DC input to a resistive load

(DC-100 MHz) current sensors are used to measure current. Pewatron differential probes are used to measure voltage. The LCL compensation network is used on primary side and series compensation network is used on secondary side. TDK MLCC capacitors are used due to its low ESR. TDK SPM6550CT type inductors are used for its high power and high frequency 

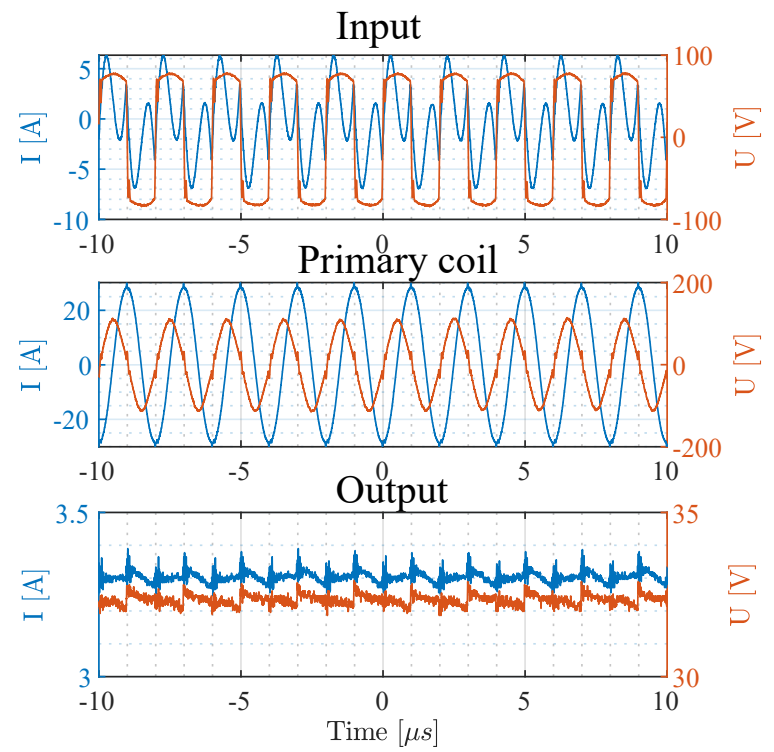

Fig. 23. Power test waveforms for coil pair 1. From top to bottom: 1. Voltage and current after full bridge inverter; 2 . Voltage and current across the primary PCB coil; 3. DC voltage and current after half bridge rectifier.

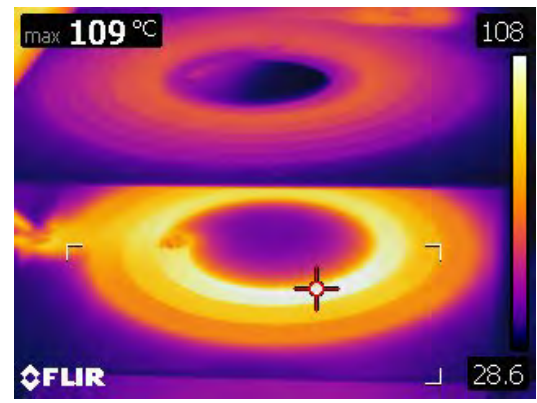

Fig. 24. Thermal image of coils in steady state.

capability.

The current and voltage waveforms for this IPT system is shown in Fig. 23. With an input DC voltage of $80 \mathrm{~V}, 34 \mathrm{~V}$ at output is expected, however the real output voltage is 33 $\mathrm{V}$. This could be explained by the parasitic resistance in the power loop and error tolerance of compensation components.

The thermal image in the steady-state is shown in Fig. 24. It can be seen that the hottest point appears in the innerest turn in primary coil, which corresponds to the modeling of magnetic field distribution in the coil. According to Fig. 24, the maximum temperature is $109^{\circ} \mathrm{C}$. In our design, the allowed maximum temperature is $125^{\circ} \mathrm{C}$. However, in our thermal model average temperature is calculated instead of highest temperature and the expected average temperature of primary coil according to (20) is $99{ }^{\circ} \mathrm{C}$.

Test under different load conditions is executed. As shown in Fig. 25, the output voltage decreases as output power increases. Load condition influences the output voltage and this is because the non-zero coil resistance would share the secondary induced voltage with load. In the no load condition, the rectifier on the secondary side is open circuit. The reflected resistance of the load to the primary side is now zero, and

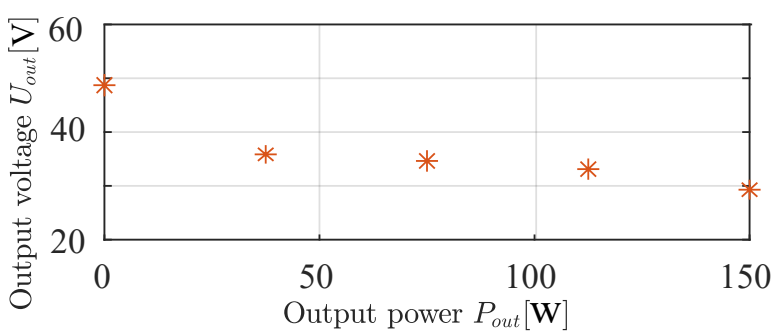

Fig. 25. Output voltage under various load condition for coil pair 1 with 60 $\mathrm{mm}$ air gap between primary and secondary coil. The load condition changes from no load condition to $150 \mathrm{~W}$.

therefore the primary current increases. The induced secondary voltage increases accordingly. The current prototype could handle the no-load condition and its minimum load requirement is defined by the insulation voltage on the secondary coil. Above $25 \%$ load $(37.5 \mathrm{~W})$, the voltage variation is within 6 $\mathrm{V}$, the voltage slope is more or less flat.

In conclusion, a coil prototype considering the application of auxiliary power supply in MV converters is tested with $80 \mathrm{~V} \mathrm{DC}$ input, $10 \Omega$ load, $60 \mathrm{~mm}$ air gap between primary and secondary coils. $33 \mathrm{~V} 110 \mathrm{~W}$ is obtained at output. The efficiency of the entire IPT system is $67 \%$ including loss on the front and back power electronics converters and loss on compensation components. Further optimization will be done to improve these numbers.

\section{CONCLUSION}

This paper focuses on the PCB coil design in IPT system for MV applications with medium power transfer capability and strict insulation requirements. The main contributions of this paper are:

Firstly, an improved magnetic field distribution model for coils with rectangular cross-section offers a fast calculation approach. The model is more accurate than the filament method.Secondly, the proposed model for self and mutual inductance and resistance can be used to do fast characterization of PCB coils. The accuracy of the model is especially high for trace width below $10 \mathrm{~mm}$. Thirdly, by doing analysis in coil geometries we found $\frac{s}{w}$ and coil inner radius shouldn't be too large otherwise coil quality factor would be low. Finally, by simply setting the design specifications and constraints, the proposed design approach could assist users with developed filters, allowing intuitive and easy navigation through a broad design space with high design freedoms. As a demonstration, a coil pair capable to transfer $110 \mathrm{~W}$ via $60 \mathrm{~mm}$ air gap is designed and prototyped.

Further ongoing design optimization works are related to optimization of IPT coil link as well as optimization in the power electronics converters and compensation networks for higher efficiency.

\section{REFERENCES}

[1] J. Dai and D. C. Ludois, "A Survey of Wireless Power Transfer and a Critical Comparison of Inductive and Capacitive Coupling for Smal Gap Applications," IEEE Transactions on Power Electronics, vol. 30, no. 11 , pp. 6017-6029, Nov. 2015. 
[2] M. Schormans, V. Valente, and A. Demosthenous, "Practical Inductive Link Design for Biomedical Wireless Power Transfer: A Tutorial," IEEE Transactions on Biomedical Circuits and Systems, vol. 12, no. 5, pp. 1112-1130, Oct. 2018

[3] M. Zargham and P. G. Gulak, "Maximum Achievable Efficiency in Near-Field Coupled Power-Transfer Systems," IEEE Transactions on Biomedical Circuits and Systems, vol. 6, no. 3, pp. 228-245, Jun. 2012

[4] P. Si, A. P. Hu, S. Malpas, and D. Budgett, "A Frequency Control Method for Regulating Wireless Power to Implantable Devices," IEEE Transactions on Biomedical Circuits and Systems, vol. 2, no. 1, pp. 22 29, Mar. 2008.

[5] S. Y. Choi, B. W. Gu, S. Y. Jeong, and C. T. Rim, "Advances in Wireless Power Transfer Systems for Roadway-Powered Electric Vehicles," IEEE Journal of Emerging and Selected Topics in Power Electronics, vol. 3, no. 1, pp. 18-36, Mar. 2015.

[6] E. S. Lee, B. G. Choi, M. Y. Kim, and S. H. Han, "Optimal Number of Turns Design of the IPT Coils for Laptop Wireless Charging," IEEE Access, vol. 9, pp. 19548-19561, 2021.

[7] K. Kusaka, M. Kato, K. Orikawa, et al. "Galvanic isolation system for multiple gate drivers with inductive power transfer \&\#x2014; Drive of three-phase inverter," in 2015 IEEE Energy Conversion Congress and Exposition (ECCE), Montreal, QC, Canada: IEEE, Sep. 2015, pp. $4525-4532$.

[8] C. Marxgut, J. Biela, J. W. Kolar, R. Steiner, and P. K. Steimer, "DCDC converter for gate power supplies with an optimal air transformer," in 2010 Twenty-Fifth Annual IEEE Applied Power Electronics Conference and Exposition (APEC), Palm Springs, CA: IEEE, Feb. 2010 , pp. 1865-1870.

[9] K. Lee and D. Cho, "Diversity Analysis of Multiple Transmitters in Wireless Power Transfer System," IEEE Transactions on Magnetics, vol. 49, no. 6, pp. 2946-2952, Jun. 2013.

[10] M. J. Chabalko and A. P. Sample, "Three-Dimensional Charging via Multimode Resonant Cavity Enabled Wireless Power Transfer," IEEE Transactions on Power Electronics, vol. 30, no. 11, pp. 6163-6173, Nov. 2015.

[11] M. Abou Houran, X. Yang, and W. Chen, "Magnetically Coupled Resonance WPT: Review of Compensation Topologies, Resonator Structures with Misalignment, and EMI Diagnostics," Electronics, vol. 7, no. 11, p. 296, 11 Nov. 2018.

[12] G. R. Nagendra, G. A. Covic, and J. T. Boys, "Determining the Physical Size of Inductive Couplers for IPT EV Systems," IEEE Journal of Emerging and Selected Topics in Power Electronics, vol. 2, no. 3, pp. 571-583, Sep. 2014.

[13] R. Bosshard, U. Iruretagoyena, and J. W. Kolar, "Comprehensive Evaluation of Rectangular and Double-D Coil Geometry for $50 \mathrm{~kW} / 85$ kHz IPT System," IEEE Journal of Emerging and Selected Topics in Power Electronics, vol. 4, no. 4, pp. 1406-1415, Dec. 2016.

[14] R. Bosshard, J. Mühlethaler, J. W. Kolar, and I. Stevanović, "Optimized magnetic design for inductive power transfer coils," in 2013 Twenty-Eighth Annual IEEE Applied Power Electronics Conference and Exposition (APEC), Mar. 2013, pp. 1812-1819.

[15] S. S. Mohan, "The design, modeling and optimization of on-chip inductor and transformer circuits," p. 215 ,

[16] K. Sun, Y. Xu, J. Wang, R. Burgos, and D. Boroyevich, "Insulation Design of Wireless Auxiliary Power Supply for Medium Voltage Converters," IEEE Journal of Emerging and Selected Topics in Power Electronics, pp. 1-1, 2020.

[17] S. Mohan, M. del Mar Hershenson, S. Boyd, and T. Lee, "Simple accurate expressions for planar spiral inductances," IEEE Journal of Solid-State Circuits, vol. 34, no. 10, pp. 1419-1424, Oct. 1999.

[18] I. Lope, C. Carretero, J. Acero, R. Alonso, and J. M. Burdío, "AC Power Losses Model for Planar Windings With Rectangula Cross-Sectional Conductors," IEEE Transactions on Power Electronics, vol. 29 , no. 1 , pp. 23-28, Jan. 2014.

[19] O. C. Spro, P. Lefranc, S. Park, et al., "Optimized Design of Multi$\mathrm{MHz}$ Frequency Isolated Auxiliary Power Supply for Gate Drivers in Medium-Voltage Converters," IEEE Transactions on Power Electronics, vol. 35, no. 9, pp. 9494-9509, Sep. 2020.

[20] K. van Schuylenbergh and R. Puers, Inductive Powering: Basic Theory and Application to Biomedical Systems, ser. Analog Circuits and Signa Processing. Springer Netherlands, 2009.

[21] X. Du and D. Dujić, "Inductive Power Transfer System with Series Connected Primary and Independent Secondary Coils," in IECON 2020
The 46th Annual Conference of the IEEE Industrial Electronics Society, Oct. 2020, pp. 3901-3906.

[22] IEC 61800-5-1: Adjustable speed electrical power drive systems - Part 5-1: Safety requirements - Electrical, thermal and energy.

[23] S. Babic and C. Akyel, "New analytic-numerical solutions for the mutual inductance of two coaxial circular coils with rectangular cross section in air," IEEE Transactions on Magnetics, vol. 42, no. 6 , pp. 1661-1669, Jun. 2006.

[24] W. Kuhn and N. Ibrahim, "Analysis of current crowding effects in multiturn spiral inductors," IEEE Transactions on Microwave Theory and Techniques, vol. 49, no. 1, pp. 31-38, Jan. 2001.

[25] R. Bosshard, J. W. Kolar, J. Mühlethaler, I. Stevanović, B. Wunsch, and F. Canales, "Modeling and $\eta-\alpha$ - Pareto Optimization of Inductive Power Transfer Coils for Electric Vehicles," IEEE Journal of Emerging and Selected Topics in Power Electronics, vol. 3, no. 1, pp. 50-64, Mar. 2015.

[26] M. Q. Nguyen, Z. Hughes, P. Woods, Y. Seo, S. Rao, and J. Chiao, "Field Distribution Models of Spiral Coil for Misalignment Analysis in Wireless Power Transfer Systems," IEEE Transactions on Microwave Theory and Techniques, vol. 62, no. 4, pp. 920-930, Apr. 2014.

[27] E. B. Rosa and F. W. Grover, "Formulas and tables for the calculation of mutual and self-inductance," p. 237 ,

[28] M. Mogorovic and D. Dujic, "100 kW, 10 kHz Medium-Frequency Transformer Design Optimization and Experimental Verification," IEEE Transactions on Power Electronics, vol. 34, no. 2, pp. 16961708, Feb. 2019.

[29] C. Zierhofer and E. Hochmair, "Geometric approach for coupling enhancement of magnetically coupled coils," IEEE Transactions on Biomedical Engineering, vol. 43, no. 7, pp. 708-714, Jul. 1996.

[30] 2221A: Generic Standard on Printed Board Design - IPC Store.

[31] S. Mehri, A. C. Ammari, J. Slama, and M. Sawan, "Minimizing printed spiral coil losses for inductive link wireless power transfer," in 2016 IEEE Wireless Power Transfer Conference (WPTC), May 2016, pp. 14

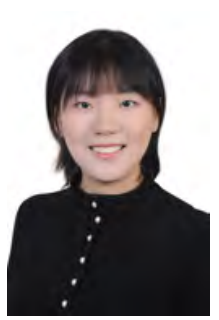

Xiaotong Du (S'19) received the B.Sc. and M.Sc degrees from Xi'an Jiaotong University, Xi'an, China, respectively in 2016 and 2019. During the B.Sc. degree, she spent two years at École Centrale de Nantes, Nantes, France, as an double degree program student and received Dipl.-Ing degree. She is currently working toward the Ph.D. degree at École Polytechnique Fédérale de Lausanne (EPFL), Lausanne, Switzerland.

In 2019, she joined Power Electronics Laboratory, EPFL, as a Doctoral Research Assistant. Her research interests include modeling, design, and optimization of inductive power transfer and resonant converters.

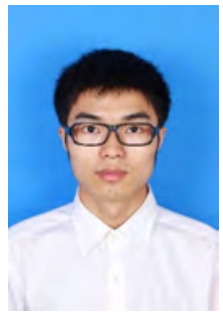

Chengmin Li (S'15-M'20) received the B.S. degree in electrical engineering from the School of Electrical and Electronic Engineering, Huazhong University of Science and Technology, Wuhan, China, in 2013. He received the Ph.D. degree from Zhejiang University, Hangzhou, China in 2019. From March 2016 to March 2017, he was a Research Intern with the GE Global Research Center, Shanghai, China. Since 2020, he has been a Postdoctoral Researcher with Power Electronics Laboratory, EPFL, Lausanne, Switzerland.

His research interests include medium voltage high-power converters and applications of $\mathrm{SiC}$ power MOSFETs. 


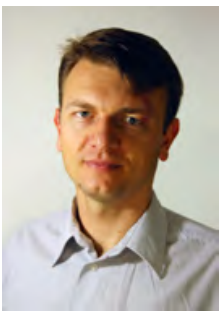

Dražen Dujić (S'03-M'09-SM'12) received the Dipl.-Ing. and M.Sc. degrees from the University of Novi Sad, Novi Sad, Serbia, in 2002 and 2005, respectively, and the Ph.D. degree from Liverpool John Moores University, Liverpool, U.K., in 2008, all in electrical engineering. From 2002 to 2006, he was with the Department of Electrical Engineering, University of Novi Sad, as a Research Assistant. From 2006 to 2009, he was with Liverpool John Moores University, as a Research Associate. From 2009 to 2013, he was with the ABB Corporate Research Centre, Switzerland, as the Principal Scientist, working on the power electronics projects spanning the range from low-voltage/power SMPS in below kilowatt range to medium voltage high-power converters in a megawatt range. From 2010 to 2011, he was a member of a project team responsible for the development of the world's first power electronic traction transformer successfully commissioned on the locomotive. From 2013 to 2014, he was with ABB Medium Voltage Drives, Turgi, Switzerland, as a Research and
Development Platform Manager, responsible for ABB's largest IGCT-based medium voltage drive ACS6000

He is currently with the École Polytechnique Fédérale de Lausanne (EPFL), Lausanne, Switzerland, as an Associate Professor and the Director of the Power Electronics Laboratory. He has authored or coauthored more than 200 scientific publications and has led 18 patents. His current research interests include the areas of design and control of advanced high-power electronics systems for medium voltage applications.

He has received the First Prize Paper Award from the Electric Machines Committee of the IEEE Industrial Electronics Society, in 2007. In 2014, he has received the Isao Takahashi Power Electronics Award for outstanding achievement in power electronics, and in 2018, the EPE Outstanding Service Award from the European Power Electronics and Drives Association. He is an Associate Editor of the IEEE TRANSACTIONS ON INDUSTRIAL ELECTRONICS, the IEEE TRANSACTIONS ON POWER ELECTRONICS, and the IET Electric Power Applications. 